\title{
A 3D Puzzle for Learning Anatomy
}

\author{
Bernhard Preim ${ }^{1}$, Felix Ritter ${ }^{2}$, and Oliver Deussen ${ }^{2}$ \\ 1 MeVis gGmbH, Universitätsallee 29, 28359 Bremen, Germany \\ 2 Inst. for Simulation and Graphics \\ Otto-von-Guericke-University of Magdeburg, Dept. of Computer Science \\ Universitätsplatz 2, 39106 Magdeburg, Germany \\ bernhard@mevis.de \\ \{deussen,fritter\}@isg.cs.uni-magdeburg.de
}

\begin{abstract}
We present a new metaphor for learning anatomy - the 3D puzzle. With this metaphor students learn anatomic relations by assembling a geometric model themselves. For this purpose, a 3D model is enriched with docking positions which allow objects to be connected together. As complex 3D interactions are required to compose 3D objects, sophisticated 3D visualization- and interaction techniques are included. Among these techniques are shadow generation, 3D input devices, snapping mechanisms and collision detection.

The puzzle, similar to a computer game, can be operated at different levels. To simplify the task, a subset of the geometry, e.g. the skeleton, can be correctly assembled initially. Moreover, textual information concerning the region of objects is provided, along with snapping mechanisms to support the user. With this approach we expect to motivate students to explore the spatial relations between parts of the human body.
\end{abstract}

Keywords: Anatomic atlas, metaphors for anatomy education, depthcues, 3D interaction, two-handed interaction

\section{Introduction}

The study of anatomy requires a deep understanding of the complex spatial relations inside the human body. With interactive 3D computer graphics, based on high resolution geometric models, these spatial relations may be explored. To exploit this potential, dedicated 3D interaction- and visualization techniques as well as convincing metaphors have to be developed. To date, most of the available systems for learning anatomy are based on the atlas-metaphor: Students explore geometric models and related textual information in a way inspired by a (printed) atlas. The leading example is the Voxel-Man [2]; another more recent system is the Zoom Illustrator [8]. The atlas metaphor does not lend itself to the development of 3D interaction techniques. Nevertheless 3D interaction is provided to a certain extent. The Voxel-Man allows the user to rotate geometric models, to place cutting planes and to cut holes. However, students are often unaware of these possibilities. 
Therefore it is particularly useful to structure the user interface on the basis of a spatial metaphor and to provide tasks which necessarily include 3D interaction. In this paper, we introduce the metaphor of a 3D puzzle for learning systems in medicine: users compose geometric models from anatomic objects themselves. This idea arose out of an empirical evaluation of the Zoom Illustrator with 12 medical doctors and students of medicine [6]. As part of a study of the usefulness of the available features, we asked what features they would like to see included in the system. Several students expressed a desire for more powerful 3D interaction.

To enable students to compose geometric models themselves, is a challenging task. Students must be able to sort geometric objects, to compose subsets before composing the whole model. Interaction- and visualization techniques which communicate depth relations play a key role in the usefulness of such a system.

\section{Metaphors for the Composition of 3D Models}

Interactive systems, especially new and unfamiliar applications, should be based on metaphors which help developers to structure the design and help users to handle the system. Metaphors should have their origin in daily life or in the work environment of the intended users.

The Construction-kit Metaphor. This wide-spread metaphor is used mainly in advanced CAD-systems. The design of cars, for example, is based on various CAD-models from different sources which are assembled into virtual prototypes using sophisticated 3D interaction techniques. An interesting variant was developed in the VLEGO-project [4]. Users take primitives, like LEGO-bricks, and combine them at discrete, predefined positions and angles. Dedicated 3D widgets are provided for all 3D interaction tasks: composition, separation, picking and copying. These 3D widgets can be handled with a $3 \mathrm{D}$ input device and for some interaction tasks a two-handed interaction is suggested.

The Metaphor of a 3D Puzzle. The construction kit-metaphor is wellknown, and the 3D interaction techniques designed in the context of this metaphor are desirable for learning anatomy. However, building blocks in construction kits are not unique. In the learning context, we have unique parts which can be assembled in only one correct manner. Therefore a metaphor is required for the composition of complex models from unique elements.

A 3D puzzle is a familiar concept for this task and consequently the puzzle metaphor is more appropriate. This raises a question: which aspects of a 3D puzzle can and should (from a user's point of view) be realized? Moreover, we have to decide what we can offer over and above mimicking the metaphor. In a puzzle, a set of elementary objects should be composed. The shape of these objects gives an indication as to which parts belong together. When puzzling with dozens or even hundreds of objects several deposits (e.g. tables) are used to sort and compose subsets. Obviously, when puzzling one uses both hands and has all DOF of spatial interaction. In a puzzle, photos are provided to show how the final composed image (or 3D model) looks. These images motivate users and 
help them to perform the composition. Our design is guided by the metaphor of a $3 \mathrm{D}$ puzzle but differs in two major respects to real puzzles:

- Our system should support learning rather than provide entertainment.

- A computer system is restricted as to what can be achieved in real time but offers additional possibilities in that the computer "knows" how the model should be assembled.

To support learning, we have incorporated textual information about the objects of the puzzle. Objects have names, belong to regions and organ systems (e.g. an eye muscle) and have textual explanations as to their shape. This information may be exploited in order to place objects in the right position. Since the system "knows" how the model is composed snapping mechanisms may be activated.

\section{Interaction Tasks with a 3D Puzzle}

In this section we describe the tasks which need to be accomplished in order to realize the metaphor of a 3D puzzle. Actually, there are two kinds of users:

- authors who prepare models (segment the model or refine an existing structure, define docking positions and assign related textual information) and

- students who use the information space provided by the author for the puzzle.

In this paper we restrict ourselves to describing how students explore the information space and assume that it is carefully defined by an author. For students some typical interaction tasks include:

Sort objects. The student must be able to create and manage subsets of the total set of objects. These subsets should be placed in separate viewers which can be named by the user. Within these viewers, $3 \mathrm{D}$ interaction is required to enable users to explore this subset. As not all viewers are visible at the same time, an overview about existing viewers is crucial.

Recognition of 3D objects. Two factors are crucial for the identification of objects: to be able to see an object from all viewing angles and to be able to inspect textual information as to spatial relations (e.g. name, description of shape). From our experience [7] we hypothesize that visual and textual information mutually reinforce one another in their effect upon the viewer.

Selection of $3 \mathrm{D}$ objects. The selection of $3 \mathrm{D}$ objects is the prerequisite for $3 \mathrm{D}$ interaction. Picking, typing the object name and the choice from a list of names are possible interaction techniques for this task.

Transformation of 3D objects. The transformation task includes translating and rotating $3 \mathrm{D}$ objects. As the objects are not deformable, transformations like shearing are irrelevant.

Camera control. In all viewers pan-and-zoom functionality is required to be able to recognize the shape of individual objects.

Docking of objects. The final goal of exploring, selecting and transforming a set of $3 \mathrm{D}$ objects is to assemble objects at the "right" docking positions. Less obvious is that objects sometimes have to be separated. For instance, if objects 
in deeper layers must be assembled first but have been forgotten, objects in the outer areas may have to be decomposed to allow objects to be placed inside.

\section{Visualization of and Interaction with 3D data}

A 3D puzzle requires precise interaction in $3 \mathrm{D}$ and thus the simulation of depthcues and 3D interaction techniques similar to those in the real world. Humans perceive depth-relations particularly from the following depth-cues [10]:

- shadow,

- occlusion of objects,

- partial occlusion of semi-transparent objects,

- perspective foreshortening, and

- stereoscopic viewing.

Some of these depth-cues, such as occlusion and perspective foreshortening, are part of standard renderers and are implemented in hardware. Shadow generation is usually not supported. In an evaluation, Wanger et al. [9] demonstrated that shadow cast on a groundplane is the most important depth-cue for distance estimation and shape recognition. Therefore we developed a shadow viewer which enables the shadow projection on a groundplane. On graphics workstations with hardware-based alphablending, the display of semi-translucent objects and stereoscopic viewing is also feasible in real-time.

Interaction with $3 D$ Data On the base of a comprehensible rendition of objects, $3 \mathrm{D}$ interaction is possible. The design of 3D interaction techniques must take into account how humans interact in the real world. The following aspects are essential for interaction in the real world:

Collision. When one object touches another, it is moved away or will be deformed. Under no circumstances can one object be moved through another without deformation.

Tactile feedback. When we grasp an object we perceive tactile feedback which enables us to adapt the pressure to the material and weight of the object.

Two-handed interaction. People tend to use both hands if they manipulate 3D objects. In medicine, two-handed interaction has been successfully applied e.g. for pre-operative planning in neurosurgery (see Hinckley in [1]). Hinckley argues that for the interaction tasks involved (e.g. defining cross-sections with cutting planes), the most intuitive handling can be achieved with two-handed 3D interaction where the dominant hand (usually the right hand) does finepositioning relative to the non-dominant hand.

We regard collision detection as the most important aspect of 3D interaction. However, this is a challenging task if complex non-convex objects are involved. Fortunately, software for this purpose is now available. The system V-COLLIDE [3] accomplishes this task in a robust manner. Tactile feedback requires special hardware, such as data gloves or joysticks with force feedback. To avoid the overhead with an unfamiliar input-device, we have not integrated this technique. 


\section{The Realization of a 3D-Puzzle}

The 3D puzzle incorporates the visualization and interaction techniques described in Section 4. In addition, some techniques from technical and medical illustration have been added to further improve the understanding of spatial relations. Our prototype is based on polygonal models (30.000 to 60.000 polygons segmented into 40 to 60 objects) acquired from Viewpoint Datalabs. The software is written in $\mathrm{C}++$ using Open Inventor and Open GL.

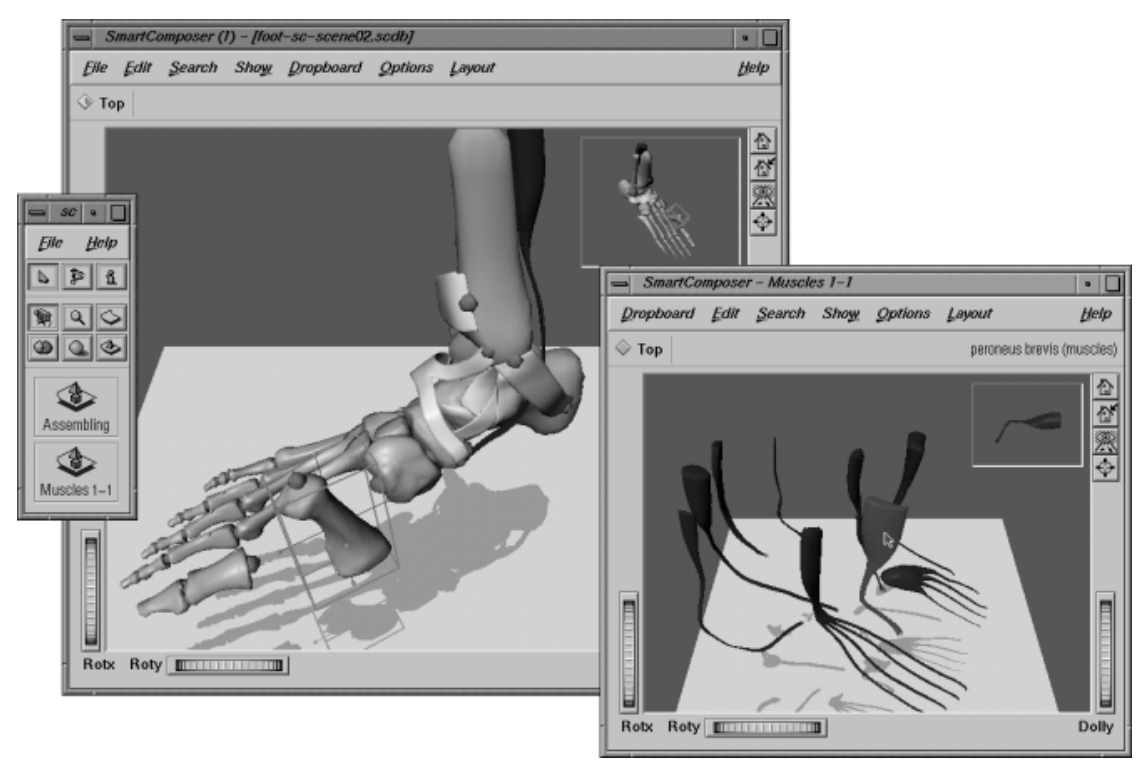

Fig. 1. In the left view sinews and bones are composed, while in the right view muscles are randomly scattered. The small panel on the left provides an overview on all viewers.

The puzzle starts with three views: the final view where the whole model is displayed, the construction view in which the user composes the model (starting from scratch or a subset of the model), and a random view in which objects which do not belong to the construction view are randomly scattered. The initial position of the objects is adjusted such that they do not overlap (see Fig. 1).

\subsection{Realization of the Interaction Tasks}

Sort objects. For the management of the objects, subsets can be created and attached to an unlimited number of $3 \mathrm{D}$ viewers. For this purpose a multiple selection of objects is possible. In addition, all objects in a region or an organsystem might be selected. The command "create view" opens a new viewer 
and moves all selected objects to this viewer while the relative position of the objects is preserved. An overview with icons for all viewers is presented to enable switching between the viewers (recall Fig. 1). While the final view is read-only, objects can be exchanged between the other views by drag-and-drop (objects may be dropped either in the viewers or the corresponding icon in the overview).

Recognition of objects. To enable the recognition of objects, we developed a shadow viewer with a light groundplane. This plane is scaled such that all objects cast a shadow on it, even if the camera is rotated (the plane remains fix as it is used only for orientation). To further enhance the recognizability of an object, we provide a detailed view, like an inset in technical illustrations. If an object is selected it is presented in this view slightly enlarged without any object occluding it. It is rotated automatically to faciliate recognition.

In technical illustrations, exploded views are provided to improve the recognizability of objects. This technique is employed in the final view to enable users to become familiar with the spatial relations. Exploded views are realized by scaling down all objects at their original positions, thus leaving empty space. Stereorendering is realized as an extension of the Silicon Graphics X-Server and requires the use of shutter glasses to perceive the rendition as a stereo image.

Selection of objects. Selection by picking with a pointing-device is the interaction inspired by the real 3D puzzle. Picking is useful but limited to objects which are visible and recognizable. Therefore, selection by name and from a list are also provided. As typing long anatomic names is tedious, an auto-complete mechanism is employed to expand names. When one of these textual interaction techniques is used, the selected object will be highlighted to provide feedback. If the object belongs to a viewer currently occluded it is sent to the front to make it visible. Moreover, the object might be occluded within its view. If this is the case, it is moved continuously towards the viewer until it is in front of other objects. To further improve selection, semi-transparency can be used, so that all objects except the one selected by name are semi-translucent.

Transforming objects. For the transformation of 3D objects, manipulators (the trackball and the handlebox) from Open Inventor are used. These widgets can be operated with a $2 \mathrm{~d}$ mouse. However, with a standard $2 \mathrm{~d}$ mouse users tend to decompose $3 \mathrm{D}$ translations and rotations in sequential $2 \mathrm{~d}$ translations. It is more effective to use several DOF simultaneously as in the real world. For this purpose a $3 \mathrm{D}$ space mouse is employed. Collision detection prevents objects from being moved through others. When objects collide they are highlighted for a moment to provide visual feedback. If the user continues to attempt to move an object through another one, an acoustic signal is initiated and textual output is provided in the status line We incorporated the above-mentioned software V-COLLIDE for collision detection. With a variety of acceleration techniques, including hierarchical decomposition of geometric models and bounding box tests, it is reasonably fast. V-COLLIDE provides an interface which allows us to control precisely for which objects the test is carried out.

Camera control. The virtual camera can be manipulated with the widgets provided by Open Inventor. Wheel-widgets make it possible to change azimuth- 
and declination angle and to zoom in and out. Camera control can be realized by two-handed interaction intuitively by simultaneously rotating and zooming.

Composing and separating objects. The composition is the most challenging task. Objects are composed correctly if the docking points (represented as spheres) touch each other. To ease this task, a snap-mechanism is included (see Fig. 2). With snapping enabled, objects snap together if their distance is below a threshold and no other docking point is in the vicinity. If docking points are very close to each other snapping does not help. In this case, the user may select the docking position to which the selected object should be attached. Once an object is correctly attached it is prevented that these objects are separated inadvertently. With a quick movement, however, separation is possible. Reverse snapping makes it difficult to attach an object to a wrong docking position.



Fig. 2. A bone has been snapped at one docking point. The transformation is now restricted to the rotation to correctly orient this bone.

\subsection{Two-handed Interaction}

The 3D puzzle supports the simultaneous use of two input devices. The optimal configuration is a $3 \mathrm{D}$ mouse for $3 \mathrm{D}$ interaction and a $2 \mathrm{~d}$ mouse for other interactions (selection from lists and the menu). For this purpose two cursors are provided; a 3D cursor for 3D interaction in addition to the usual cursor. People use their dominant hand $(\mathrm{DH})$ for interaction with menus and dialogue boxes. Therefore the $2 \mathrm{~d}$ mouse is usually operated with the $\mathrm{DH}$ while the $3 \mathrm{D}$ mouse is used with the non-dominant hand (NDH). The 3D mouse is mainly used for the orientation of the camera and the 3D objetcs - orientation tasks which are carried out with the NDH also in the real world. The use of two input devices prevents the user from distractive movements from the $3 \mathrm{D}$ viewers to other components 
of the user interface and vice versa. This separation of concerns is inspired by Leblanc [5]. Informal tests indicate that users have superior performance with this equipment compared to the standard pointing device.

\subsection{Adapting the Level of Complexity}

A learning system should be operable at different levels. Usually interactive systems should be as easy to operate as possible. However, with the $3 \mathrm{D}$ puzzle it should take some time to succeed because the time spent on solving this task is probably related to the learning success. On the other hand, users might become frustrated if it is too difficult to succeed. There are two strategies to adapt the level: to "scale" the task to be solved, and to provide support for solving the task. The easiest way to use the system is to watch the model being assembled in an animation, so the user has no task at all. The composition can be restricted to objects of certain categories (e.g. bones) and regions (e.g. eye muscles). The composition can be performed at two levels. At the beginners level, objects are rotated correctly when they are dropped to the construction view. The task is thus restricted to the correct translation of the object. The display of textual information for a selected object (e.g. Musculus procerus, eye muscle) and the mechanisms for snapping and reverse snapping (recall Section 5.1) provide support for the puzzle.

\section{Summary}

We presented a system for anatomy education based on the metaphor of a 3D puzzle. With this metaphor users have a precise task involving spatial relations. The puzzling task provides a level of motivation for learning which is hard to achieve with other metaphors. The metaphor of a 3D puzzle guided our design and led us to incorporate advanced visualization and interaction techniques to enable students to compose 3D models. Different levels are provided to acommodate users with different capabilities. The development of our system has been accompanied by informal usability tests which yielded promising results. We intend to perform a rigorous usability test. In particular, the use of twohanded interaction, the snapping mechanisms and the different levels will be evaluated. With regard to the interaction, tactile feedback offers great promise as an addition to collision detection.

\section{References}

1. Hinckley, K. (1997): Haptic Issues for Virtual Manipulation, PhD-thesis, University of Virginia 753

2. Höhne, K.-H., B. Pflesser, A. Pommert et al. (1996): "A Virtual Body Model for Surgical Education and Rehearsal", Computer - Innovative Technology for Professionals, January, 25-31 750 
3. Hudson, T.C., M.C. Lin, J. Cohen, S. Gottschalk and D. Manocha (1997): "VCOLLIDE: Accelerated Collision Detection with VRML", Proc. of VRML 753

4. Kiyokawa, K., H. Takemura, Y. Katayama, H. Iwasa and N. Yokoya (1997): "VLEGO: A Simple Two-handed Modeling Environment Based On Toy Block", In Proc. of VRST '97, 27-34, ACM, New York 751

5. LeBlanc, A., P. Kalra, N. Magenat-Thalmann and D. Thalmann (1991): "Sculpting with the 'ball and mouse' metaphor", Proc. of Graphics Interface, 152-159 757

6. Pitt, I., B. Preim and S. Schlechtweg (1999): "Evaluation of Interaction Techniques for the Exploration of Complex Spatial Phenomena", Proc. of Softwareergonomie 99, 275-286 751

7. Preim, B. (1998): Interaktive Illustrationen und Animationen zur Erklärung komplexer räumlicher Zusammenhänge, PhD-thesis, Otto-von-Guericke-University of Magdeburg 752

8. Preim, B., A. Ritter and Th. Strothotte (1996): "Illustrating Anatomic Models A Semi-Interactive Approach", Proc. of Visualization in Biomedical Computing, Springer publishing company, Lecture Notes in Computer Science, Vol. 1131, 23-32 750

9. Wanger, L., J. Ferwerda and D. Greenberg (1992): "Perceiving Spatial Relationships in Computer-Generated Images", IEEE Computer Graphics and Applications, Volume 12 (3), 44-58 753

10. Zhai, S., W. Buxton and P. Milgram (1996): "The partial occlusion effect: utilizing semi-transparency in 3D human computer interaction", ACM Transactions on HCI, Vol. 3 (3), 254-284 753 